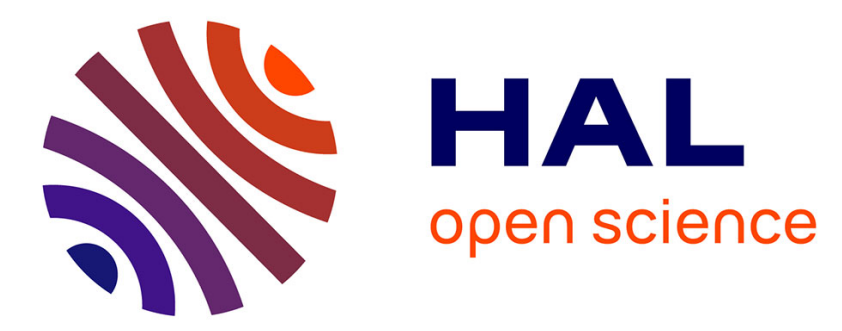

\title{
High-Throughput Monitoring of Single Vesicle Fusion Using Freestanding Membranes and Automated Analysis
}

Sathish Ramakrishnan, Andrea Gohlke, Feng Li, Jeff Coleman, Weiming Xu, James E Rothman, Frédéric Pincet

\section{- To cite this version:}

Sathish Ramakrishnan, Andrea Gohlke, Feng Li, Jeff Coleman, Weiming Xu, et al.. High-Throughput Monitoring of Single Vesicle Fusion Using Freestanding Membranes and Automated Analysis. Langmuir, 2018, 34 (20), pp.5849-5859. 10.1021/acs.langmuir.8b00116 . hal-01954048

\section{HAL Id: hal-01954048 \\ https: / hal.sorbonne-universite.fr/hal-01954048}

Submitted on 13 Dec 2018

HAL is a multi-disciplinary open access archive for the deposit and dissemination of scientific research documents, whether they are published or not. The documents may come from teaching and research institutions in France or abroad, or from public or private research centers.
L'archive ouverte pluridisciplinaire HAL, est destinée au dépôt et à la diffusion de documents scientifiques de niveau recherche, publiés ou non, émanant des établissements d'enseignement et de recherche français ou étrangers, des laboratoires publics ou privés. 


\section{High throughput monitoring of single vesicle fusion using free-standing membranes and automated analysis}

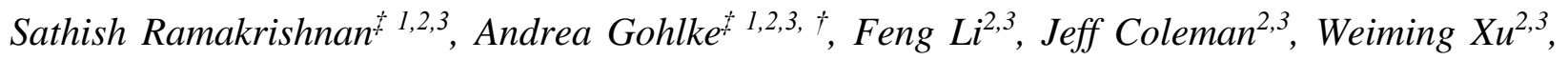
James E. Rothman ${ }^{* 2,3}$, Frederic Pincet ${ }^{* 1,2,3}$

${ }^{l}$ Laboratoire de Physique Statistique, Ecole Normale Supérieure, PSL Research University, Université Paris Diderot Sor-bonne Paris Cité, Sorbonne Universités UPMC Univ Paris 06, CNRSParis, France; ${ }^{2}$ Department of Cell Biology, Yale School of Medicine, New Haven, 333 Cedar St., Connecticut 06510, USA; ${ }^{3}$ Nanobiology Institute, 850 West Campus Drive, West Haven, CT 06516 USA.

* Correspondence to Frederic Pincet (pincet@lps.ens.fr) or James E. Rothman (james.rothman@yale.edu)

KEYWORDS: Content release, lipid mixing, holey silicon substrate, protein mobility, SNARE. 


\section{ABSTRACT}

In vivo membrane fusion primarily occurs between highly curved vesicles and planar membranes. A better understanding of fusion entails an accurate in vitro reproduction of the process. To date, supported bilayers have been commonly used to mimic the planar membranes. SNARE proteins, that induce membrane fusion, usually have limited fluidity when embedded in supported bilayers. This alters the kinetics and prevents correct reconstitution of the overall fusion process. Also, observing content release across the membrane is hindered by the lack of second aqueous compartment. Recently, a step towards resolving these issues was achieved by using membranes spread on holey substrates. The mobility of proteins was preserved but vesicles were prone to bind to the substrate when reaching the edge of the hole, preventing the observation of many fusion events over the suspended membrane. Building on this recent advance, we designed a method for the formation of pore-spanning lipid bilayers containing t-SNARE proteins on $\mathrm{Si} / \mathrm{SiO} 2$ holey chips allowing the observation of many individual vesicle fusion events by both lipid mixing and content release. With this setup, proteins embedded in the suspended membrane bounced back when they reached the edge of the hole which ensured vesicles did not bind to the substrate. We observed SNARE-dependent membrane fusion to free-standing bilayer of about 500 vesicles. The time between vesicle docking and fusion is $\sim 1 \mathrm{~s}$. We also present a new multi-modal open source software, Fusion Analyzer Software (FAS), which is required for fast data analysis. 


\section{INTRODUCTION}

Model membranes are often used to mimic physiological processes in vitro. By offering a simplified and controlled environment, they provide valuable information that would be difficult to obtain from complex in vivo systems. For decades, membrane fusion, which is an archetypal example of such process, has been well reproduced and characterized using primarily small liposomes and supported membranes as model membranes. The well-established bulk liposome/liposome fusion assay detects cumulative effects. ${ }^{1}$ It provided the initial evidence that soluble N-ethylmaleimide-sensitive factor attachment protein receptors (SNAREs) are the core of the protein machinery that fuels membrane fusion and was subsequently used in hundreds of studies to better characterize this machinery. However, observation of a single fusion event is not possible with this assay because of the low sensitivity and time resolution. Hence, other technical approaches have been envisioned. For instance, Ha's group has developed a setup in which the fusion of two tethered vesicles is evidenced by monitoring the mixing of the lipids from both membranes. ${ }^{2}$ Later on, this technique was further improved for content mixing measurements. ${ }^{3}$ In most physiological fusion processes, small vesicles fuse with flat membranes, e.g. in neurotransmission, synaptic vesicles fuse with the presynaptic plasma membrane. Supported bilayers that mimic this flat geometry were employed to study SNARE-mediated single vesicle fusion events in combination with fluorescence microscopy. ${ }^{4-6}$ However, despite being an established tool the method suffers from immobile embedded proteins, interactions with the substrate, and lack of a second aqueous compartment. The protein mobility and substrate interactions were partially resolved by the introduction of polyethylene glycol spacer between the membrane and the substrate. ${ }^{7}$ While this technique allows the observation of single vesicle fusion 
by lipid mixing, it does not permit recording of cargo release through fusion pores. Due to the number of drawbacks associated with the supported bilayers, there is a demand for an alternative method to mimic the fluid nature of the native membrane separating two distinct compartments.

Pore-spanning planar lipid bilayers provide an attractive alternate system in which cis- and transside of the membrane can be monitored separately providing a native environment for membrane proteins and lipids. Claudia Steinem's group pioneered the generation of pore-spanning membranes and showed their application towards SNARE fusion. Using confocal microscopy, the group showed SNARE-mediated single vesicle fusion events on both supported and free-standing lipid bilayers. Each cavity was large enough to spatially address the lipid bilayer on top of the substrate. ${ }^{8}$ However, major disadvantages of the reported method are that the vesicles were mostly binding at the rim of the substrate, docking times were long, docked vesicle seemed to poorly diffuse and the fluorescence readout from top of the chamber requires large aqueous volume. An improved method proposed by the same group using larger holes overcame some of these disadvantages. ${ }^{9}$ Despite a satisfactory lateral mobility of proteins, liposomes initially docked to the free-standing membrane tended to bind to the substrate upon reaching the edge of the hole. Hence, only a few percent of liposomes actually fused with the free-standing membrane. These liposomes exhibited docking times between 4 and $60 \mathrm{~s}$ before final fusion occurred, which is on average shorter than supported membranes with observed docking times up to 150 s. ${ }^{5,8,10,11}$ Since physiological fusions occur after $\sim 1 \mathrm{~s}$, the faster kinetics shows the relevance of free-standing membranes compared to supported membranes. No content release has been reported yet with pore-spanning membranes. However, since Boxer and coworkers have shown at a single vesicle level that inner leaflet lipids could mix without content release, ${ }^{12}$ it became clear that content- 
mixing experiments are crucial to derive conclusions of fusion experiments. Hence, it still remains challenging to mimic the physiologically relevant environment in an artificial system with fast fusion time scales and the ability to do content release experiments.

In addition to the development of model membrane systems, a computer-aided image analysis is required to decrease the work load in analyzing single vesicle fusion events. Currently, a majority of data analysis is based on manually defined regions of interest. However, this method is time consuming and labor intensive to analyze large data sets in particular. A previous study has used a particle tracking algorithm to speed-up data analysis. ${ }^{13}$ This method provides a good setting for analyzing lipid mixing in the vesicles, but it is more labor intensive when both lipid mixing and content release need to be tracked. Also, it adds complexity when the method is based on porespanning membranes as the grid pattern needs to be recognized by the software for automatic processing of several holes.

Here, we propose an optimized pore-spanning lipid bilayer system capable of recording SNAREmediated fusion events by lipid-mixing as well as content release at a sub $50 \mathrm{~ms}$ time scale. In this system, a freestanding lipid bilayer containing the full length transmembrane t-SNARE complex (syntaxin 1/SNAP-25) was formed by Giant Unilamellar Vesicle (GUV) spreading on a $\mathrm{Si} / \mathrm{SiO}_{2}$ chip exhibiting $5 \mu \mathrm{m}$ wide holes. Fluorescent small unilamellar vesicles (SUVs) containing vSNAREs (VAMP2) were added to this system, allowing us to monitor SNARE dependent single vesicle fusion events using confocal microscopy with a resonant scanner. Using this assay, we are not only able to follow lipid and protein diffusion in the planar membrane, vesicle docking and lipid spreading but also content mixing during the fusion process. In addition, we introduce a newly developed robust open-source analysis software, the "Fusion Analyzer Software" (FAS), that 
enables high-throughput analysis of membrane fusion recorded by fluorescence confocal microscopy. This program was mainly developed for the pore-spanning membrane system, but the source-code can be customized for other methods as well.

\section{RESULTS}

\section{Generation of pore-spanning proteo-membranes and their characterization.}

Pore spanning proteo-membranes are formed by the disruption of GUVs containing transmembrane proteins on a $\mathrm{Si} / \mathrm{SiO} 2$ chip (Micromotive, Germany). Using optical adhesive (NOA 81, Norland Optical Adhesives) eight chips are evenly placed and glued to a glass coverslip and enclosed with 8-well Ibidi sticky slide (Figure 1A (i)). Each chip contains an array of thousands of cylindrical holes ( $5 \mu \mathrm{m}$ diameter $\mathrm{x} 100 \mu \mathrm{m}$ depth) which can each hold up to $2 \mathrm{pL}$ of solution (Figure 1A (ii)). The measured dimensions of each well are $9.4 \times 10.7 \times 6.8 \mathrm{~mm}(\mathrm{w} \times 1 \times \mathrm{h})$ and a minimum volume of $300 \mu \mathrm{L}$ solution is required to cover the chip. This setup makes the recording of membranes accessible by confocal microscopy. We previously published a suitable protocol for generating proteo GUVs through osmotic shock, which was shown to be applicable to a range of transmembrane and membrane-associated proteins, allowing them to retain their functionality. ${ }^{14}$ We used this protocol to prepare proteo-GUVs. Here, a full-length t-SNARE clone containing a single cysteine (SNAP25-Q20C) was expressed and purified, labeled with Alexa-488 and reconstituted into GUVs composed of 70 mol\% 1,2-dioleoyl-sn-glycero-3-phosphocholine (DOPC) and 30 mol\% 1,2-dioleoyl-sn-glycero-3-[phospho-L-serine] (sodium salt) (DOPS) with a protein to lipid ratio of 1:1000 in a $0.2 \mathrm{mM}$ sucrose containing buffer (Figure 1B and Material and Methods for details on the protein and protocols). 
A

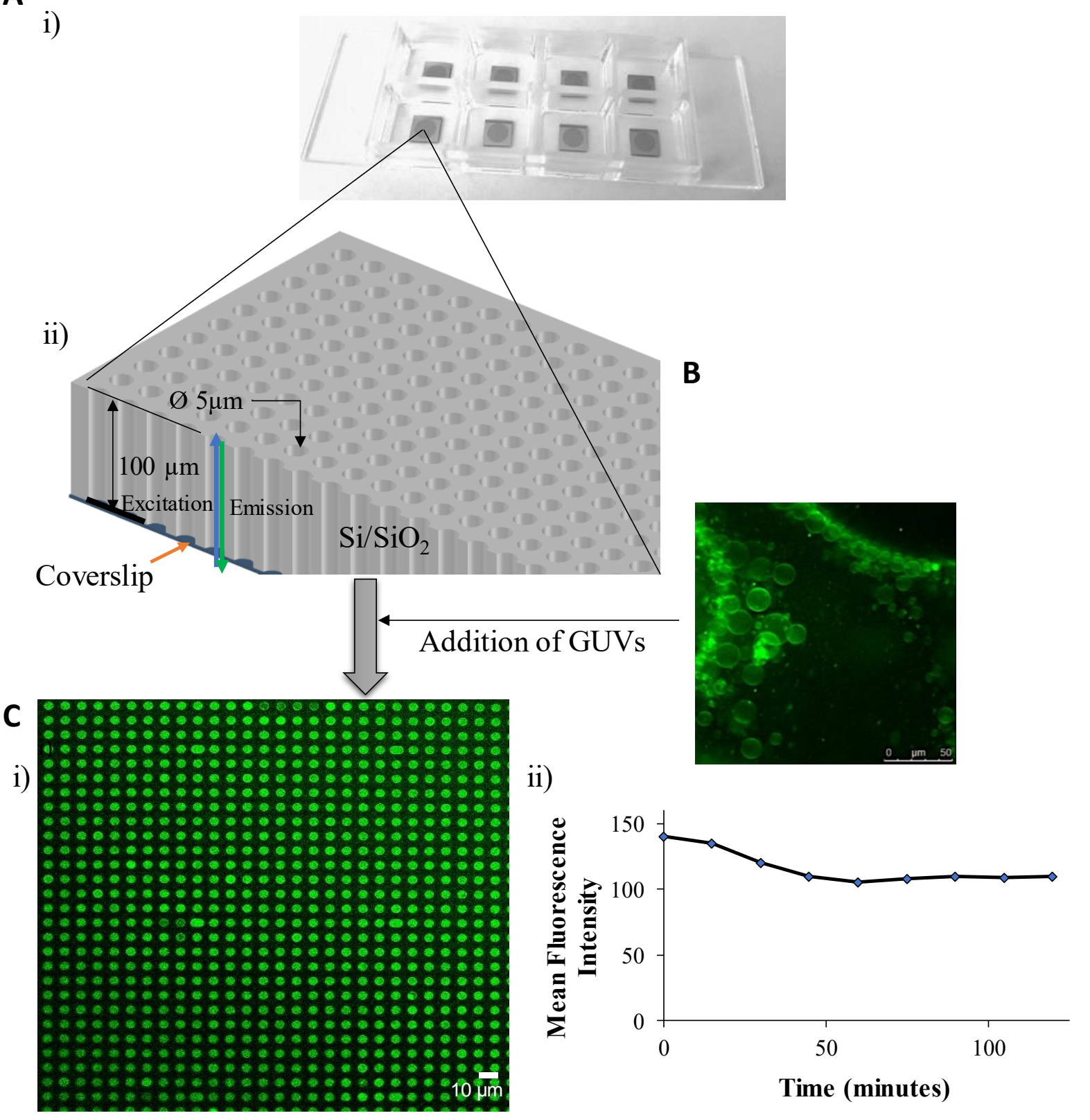

Figure 1. Pore spanning Proteo-Membrane formation on a Si/SiO2 chip A) (i) Implementation of the chip into a microscope suitable setup (ii) Schematic representation of the chip; the path of the laser beam (excitation) and emitted fluorescence (emission) are indicated in one hole at the edge of the chip B) Picture of proteo-GUVs with Alexa488 labeled transmembrane proteins ( $t$-SNAREs) during their formation with the osmotic shock protocol C) (i) Confocal image of pore spanning membranes formed by spreading proteo-GUVs containing Alexa488 labeled t-SNARE on a $\mathrm{Si}_{\mathrm{SiO}}$ chip. Fluorescence can only be seen over the hole as the substrate is opaque. Each fluorescence green dot depicts the presence of transmembrane protein above holes and attests that there is a pore-spanning membrane. (ii) Time lapse of the fluorescence signal from the pore-spanning membrane shows that the protein concentration remains constant over several hours. 
After formation, the proteo-GUVs were pipetted onto a plasma cleaned, ethanol and water/buffer rinsed $\mathrm{Si} / \mathrm{SiO} 2$ chip and centrifuged in presence of buffer $(25 \mathrm{mM}$ HEPES, $100 \mathrm{mM} \mathrm{KCl}, 1 \mathrm{mM}$ DTT, $\mathrm{pH} 7.4$ ) containing $5 \mathrm{mM} \mathrm{MgCl} 2$ to mediate the attachment and rupture of GUVs on the chip. The residual low amounts of sucrose incorporated into the proteo-GUVs lead to slow sedimentation. We found that controlled time-dependent formation of proteo-GUV is crucial to achieve maximum lipid bilayer spreading on the chip and should take about 45 mins for best results (Figure S1). Use of $5 \mathrm{mM} \mathrm{MgCl} 2$ is recommended to obtain high coverage in addition to GUV formation time. Unspread and loosely bound GUVs were removed by washing carefully with magnesium-free buffer. A homogeneous fluorescence distribution was observed across the hole showing that the incorporated proteins are spread evenly in the freestanding part of the bilayer (Figure 1C (i)). The fluorescence signal from the hole remained unchanged after 2 hours, confirming the stability of the lipid bilayer to perform time-lapse measurements (Figure $1 \mathrm{C}$ (ii)). The part of the membrane bound to the substrate (dark areas in Figure 1C (i)) is not visible because $\mathrm{Si} / \mathrm{SiO} 2$ is opaque. This protocol can be adapted to generate pore spanning membranes containing any transmembrane proteins of interest.

\section{Capturing of content beneath the pore spanning proteo membrane.}

To verify that the proteo-membranes are pore-spanning and able to separate the bottom and top compartments from each other, we added $20 \mathrm{mM}$ soluble Tetramethylrhodamine (TRITC) in the buffer prior to GUV rupture and a fluorescent lipid (NBD-DOPE) at $1.5 \mathrm{~mol} \%$ in the bilayer; proteins were not fluorescent in this experiment. After spreading of the proteo-GUV on the 
substrate, the buffer was exchanged with TRITC-free buffer. Figure 2A shows that TRITC was entrapped under the pore-spanning membranes inside the holes. A subsequent wash with $20 \%$ Sodium Dodecyl Sulfate (SDS) detergent solubilizes the lipid membrane, resulting in release of the captured fluorophore (Figure 2B). Therefore, we can conclude that our formed pore-spanning membranes are intact and are sealing the holes.

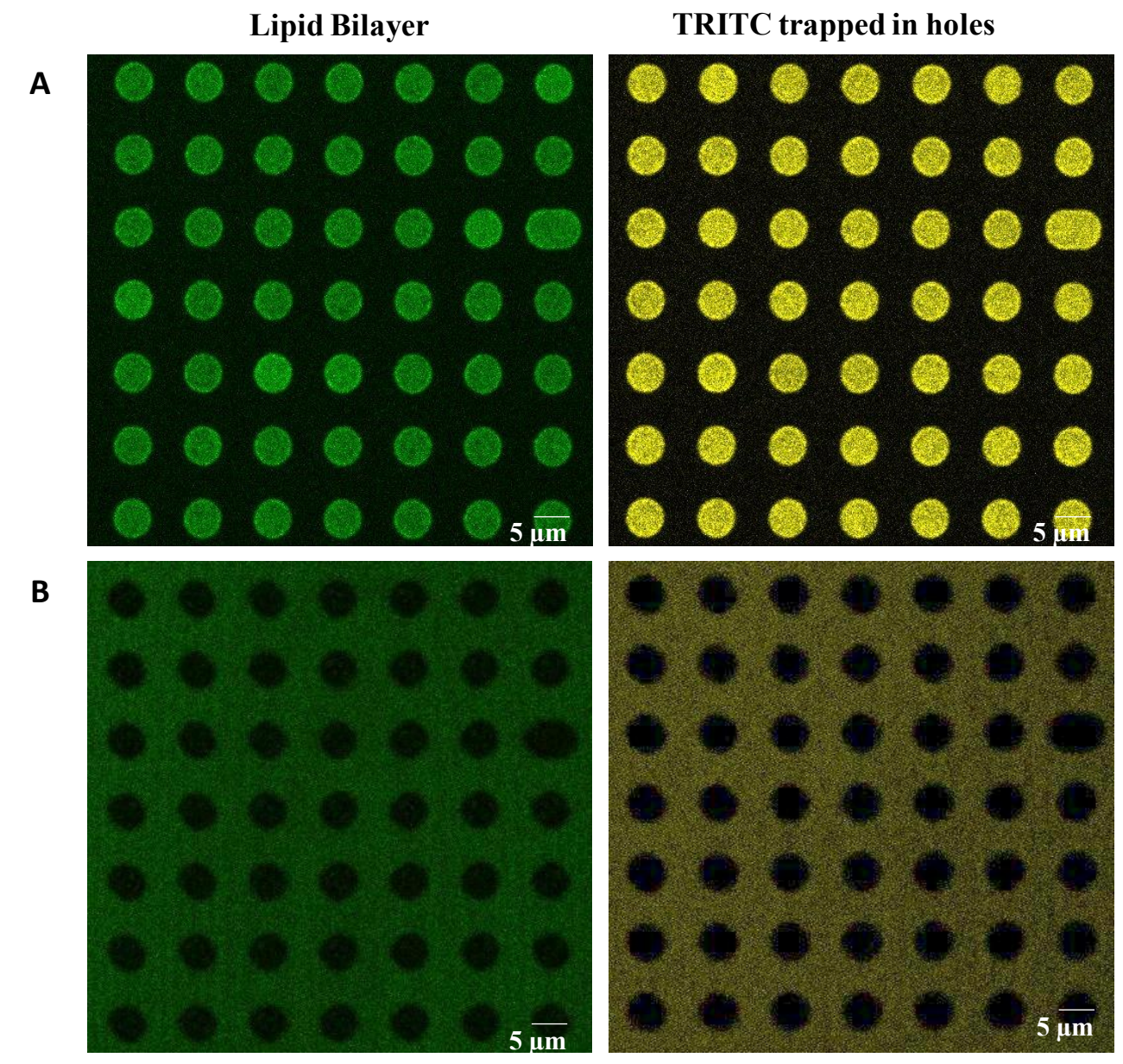

Figure 2. The pore-spanning membrane physically isolates the lower compartment from the upper one. A) Imaging of $20 \mathrm{mM}$ entrapped soluble Tetramethylrhodamine (TRITC) dye below the pore spanning membrane. The fluorescent lipids, NBD-DOPE, of the bilayer are shown in green (left) whereas the trapped soluble fluorophore is shown in yellow (right). B) After solubilizing the lipid bilayer with 20\% SDS, no fluorescence signal was observed in the lipid (left) and the trapped soluble dye (right) channel indicating that the trapped soluble dyes have diffused out of the hole. 


\section{Lamellarity of pore spanning proteo-membranes.}

A

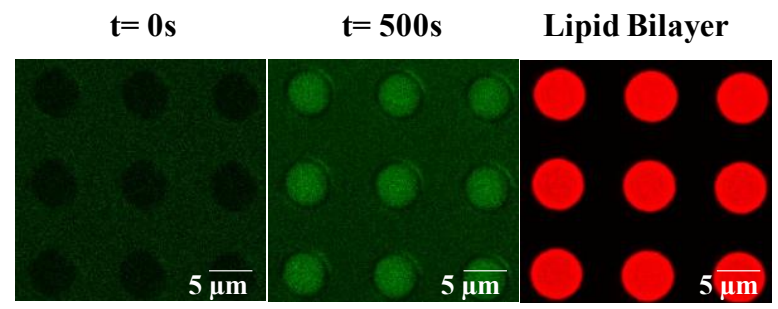

C

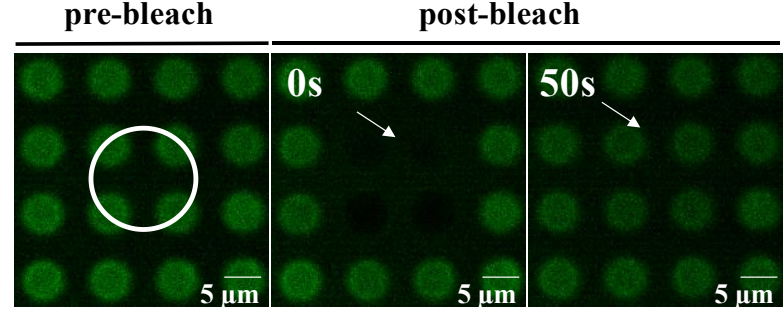

$\mathbf{E}$

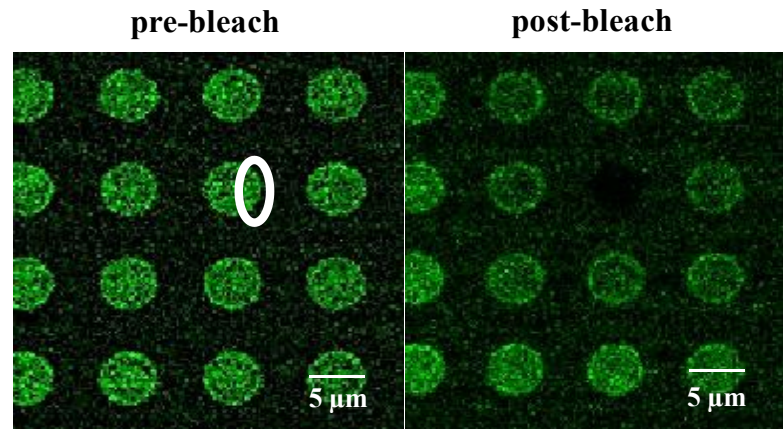

B

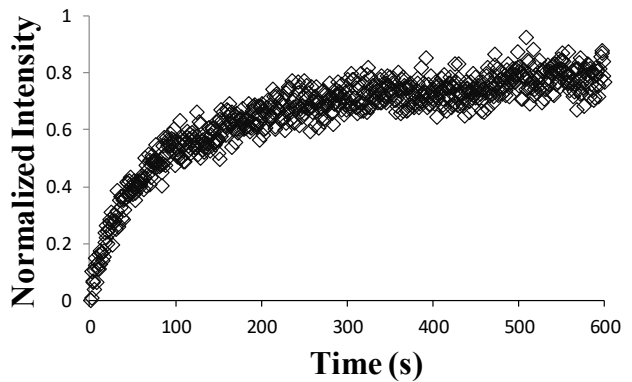

D

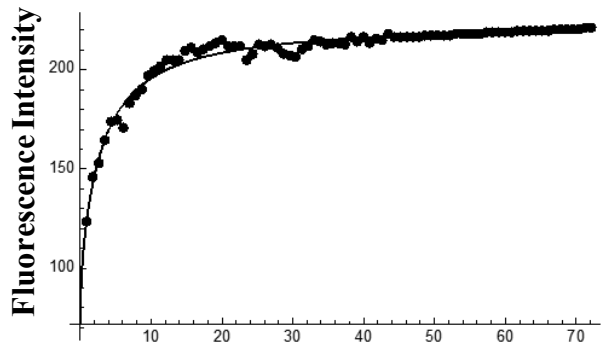

Time (s)

Figure 3. Unilamellarity and fluidity of the pore-spanning membrane. A) Images showing the influx of soluble Alexa Fluor 488 dye to the hole through $\alpha$-hemolysin pore formed on the pore-spanning membrane (Left two panels). The focus of the microscope is in the hole, several micrometers below the membrane. No fluorescence was observed at the start $(t=0 s)$ and a rapid increase in fluorescence (until $t=500 \mathrm{~s}$ ) after pore was formed demonstrates that the membranes are unilamellar. Lipid bilayer fluorescence on the right panel was imaged with ATTO647N-DOPE B) Time dependent fluorescence intensity increase of soluble Alexa488 inside the hole upon the addition of $\alpha$-hemolysin. C) Representative confocal laser scanning images showing recovery of fluorescence lipids (NBD-DOPE) after photobleaching. Complete loss of fluorescence in the holes (white circle) after photobleaching and the fluorescence recovery shows that pore-spanning membranes are continuous with appropriate fluidity. D) FRAP recovery curve of incorporated fluorescent lipid NBD-DOPE after completely bleaching a $10 \mu \mathrm{m}$ circular area encompassing one hole and part of the surrounding substrate. The recovery curve corresponds to a diffusion coefficient of $3.8 \pm 0.4 \mu \mathrm{m}^{2} / \mathrm{s}$. E) Alexa488 labelled reconstituted proteins in pore spanning membranes shown in green. Representative snapshots of pre-and post-bleached state of a hole. The white ellipse on the left panel represents the elliptical ROI that covers onethird and within which the protein fluorescence was bleached. Complete loss of fluorescence over the entire hole area indicates that all the proteins within the hole have passed through the bleaching area and is evidence of protein mobility in the pore-spanning membrane. 
To test the lamellarity of the membrane spanning over the holes, we performed experiments with a bacterial toxin, $\alpha$-hemolysin, which is well known for its ability to form pores in the lipid bilayers. It is a $33 \mathrm{kDa}$ water-soluble monomer that binds to the lipid phosphocholine head groups and spontaneously assembles on the membrane to form a $1.4 \mathrm{~nm}$ diameter heptameric transmembrane pore. ${ }^{15}$ The $\alpha$-hemolysin heptamer is a $10 \mathrm{~nm}$ long mushroom shaped complex with a transmembrane domain length of $5.2 \mathrm{~nm}$, which is nearly the size of a lipid bilayer. Existence of multilamellarity can be detected in an artificial lipid bilayer using $\alpha$-hemolysin as it cannot span over the next bilayer and thus the transport across multiple membranes is not possible. The estimated size cut off for transport of macromolecules by the pore is about $2 \mathrm{kDa} .{ }^{16}$ The final density of $\alpha$-hemolysin pores is a few dozen pores $/ \mu \mathrm{m}^{2} \cdot{ }^{17}$ After formation of the proteo-GUVs, which included $1.5 \mathrm{~mol} \%$ Atto647N-DOPE to mark the bilayer, $100 \mu \mathrm{M}$ of Alexa Fluor 488 soluble dye was added to the chip. No fluorescence signal was observed in the hole below the membrane (Figure 3A). After addition of $0.5 \mu \mathrm{M}$ of $\alpha$-hemolysin a rapid increase of Alexa Fluor 488 fluorescence signal was observed while the membranes remained intact (Figure 3B). The crossing of the membrane by the soluble dye would not be possible with multi-layers. This demonstrates that a single lipid bilayer was formed over the holes.

\section{Mobility of lipids and transmembrane proteins.}

Using fluorescence recovery after photobleaching (FRAP), we measured the lateral lipid diffusion coefficient using fluorescent lipids, dioleoyl-phosphoethanolamine-nitro-benzoxadiazolyl (NBDPE, $1 \mathrm{~mol} \%$ ), in pore spanning membranes (Figure 3C). The circular bleached area completely 
covered a single hole and was sufficiently small $(10 \mu \mathrm{m})$ to leave the fluorescence of neighboring holes intact. We recorded that fluorescence intensity was fully restored proving that the lipid molecules of both leaflets are able to migrate over the substrate and maintain continuity across the holes (Figure 3D). The obtained diffusion coefficient of $3.8 \pm 0.4 \mu \mathrm{m}^{2} / \mathrm{s}$ was calculated by computing best fit to the recovery curves, which is slightly slower than in corresponding proteoGUVs $\left(\sim 7 \mu \mathrm{m}^{2} / \mathrm{s}\right),{ }^{14}$ probably because of lipid/substrate interactions.

Mobility of lipids is not sufficient to make an in vitro membrane a good platform to mimic physiological processes. Lateral protein movement is necessary as well. The fluidity of the transmembrane protein was assessed in pore-spanning membranes made from proteo-GUVs containing Alexa488 labeled t-SNAREs. No recovery was observed upon complete bleaching of a single hole suggesting the protein cannot be exchanged between the hole and the substrate. To confirm this result and check that proteins are mobile within the pore-spanning membrane, an elliptical region of interest ( $30 \%$ of the hole surface) at the edge of the hole of the pore spanning membrane was continuously bleached for $\sim 10 \mathrm{~s}$. As a result, the fluorescence of the entire hole is bleached showing that the proteins on the pore spanning membranes are mobile, but not on the substrate, in contrast to the lipids (Figure 3E). These results indicate that the incorporated proteins are mobile across the hole and remain trapped inside a single hole, bouncing back on the rim of the substrate. Hence, each hole contains an independent pool of proteins. Proteins that interact with the substrate are likely to be almost immobile. This correlates well with supported bilayer observations and confirms the need for working with free-standing lipid bilayer to better mimic processes taking place in fluid biological membranes. The protein diffusion coefficient of the order of $1 \mu \mathrm{m}^{2} / \mathrm{s}$ was roughly estimated by pseudoquantitative measurements (Figure S4). 
To conclude the previous sections, we have developed a simple and efficient way of generating free-standing unilamellar pore-spanning membranes with integrated mobile transmembrane proteins to study processes happening in the membrane and/or transport across the membrane. Here, we have used t-SNAREs but this protocol should work with any protein that can be inserted in proteo-GUVs by, for example, the osmotic shock method. ${ }^{14}$

Using the pore-spanning membrane to monitor physiological processes - the example of SNARE-induced membrane fusion: lipid mixing and content release.

A

i)

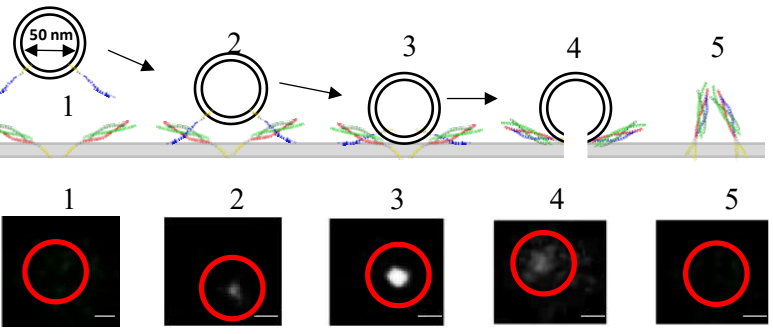

ii)

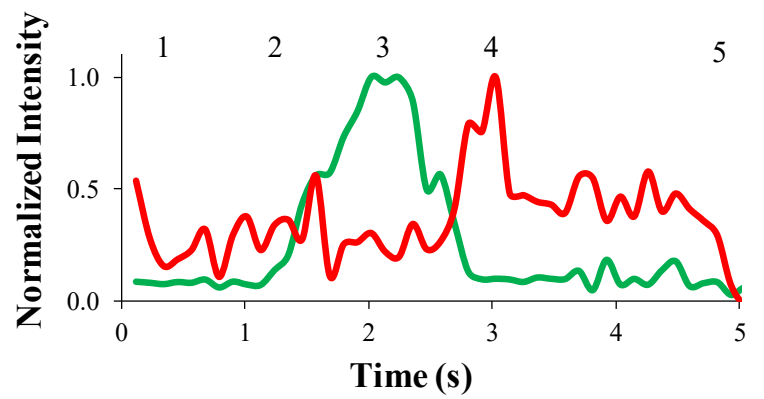

B

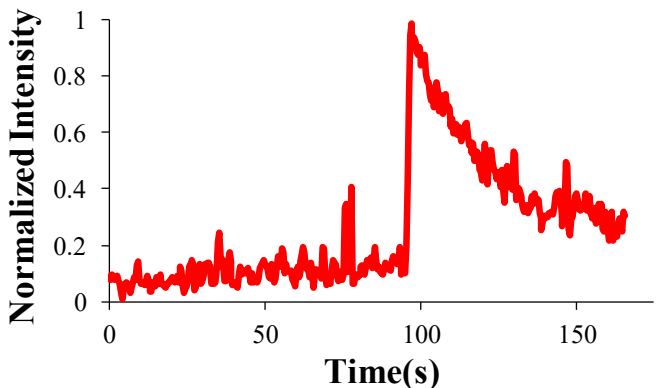

C

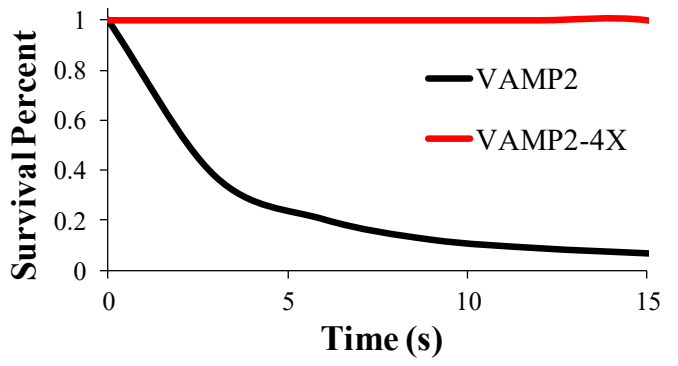

Figure 4. Lipid mixing assay A) (i) Schematic illustration of the fusion process and the corresponding recorded image sequences of the VAMP2 vesicle docked on the bilayer at different timings (Scale bar $=1 \mu \mathrm{m}$ ) (ii) Tracking of fluorescent liposomes on the pore spanning membrane. Fluorescence intensity of the vesicle was traced with an ROI (green) and the outer ROI minus the inner ROI to show subsequent lipid diffusion in the pore spanning membrane (red). B) Normalized fluorescence intensity of VAMP2-4X docked vesicles over time on the membrane undergoing photobleaching (100s) instead of fusion (1-2s). C) Survival curve of docked liposomes with v-SNAREs (VAMP2) and mutant v-SNAREs (VAMP2-4X) (Total number of vesicle analyzed, $n=500$ ). 
SNARE complexes are the core molecular machinery that drive membrane fusion, which represents the final step in many trafficking pathways. The assembly of $\mathrm{t}$ - and v-SNAREs bridges the membranes and is capable of bringing them into very close contact and eventually fusion. Upon opening of the fusion pore, the membranes are mixed and the vesicle content is released towards the other side of the membrane (Figure 4A (i)). In this part, we will show that our pore-spanning proteo-membranes provide a suitable platform to simultaneously monitor both membrane mixing and content re-lease during the fusion of a single SUV decorated with v-SNAREs (v-SUV) and a t-SNARE containing pore-spanning membrane.

The pore-spanning membrane had the same lipid composition as in the characterization experiments presented above, a DOPC:DOPS mixture (70:30), and contained Alexa488-tSNAREs (1000:1 lipid to protein ratio). v-SNARE containing SUVs (v-SUVs) were composed of DOPC/DOPS/ATTO-647N-DOPE (82:15:3) with reconstituted full-length v-SNARE, VAMP2 (400:1 lipid to protein ratio). Protein amounts and their activity after reconstitution was tested using SDS-gel electrophoresis and a bulk fusion assay (Figure S2). Note that, at this concentration, the ATTO647N dyes are initially quenched.

In some experiments, to monitor content release, calcium ions were encapsulated in the v-SUVs; Mag-Fluo-4 that was already present in the hole, beneath the bilayer, becomes fluorescent upon release of the calcium ions (see material and methods for details). v-SUVs (600 nM lipids) were added above the pore spanning membrane at $37{ }^{\circ} \mathrm{C}$. We used an inverted laser scanning confocal microscope with a resonant scanner $(16 \mathrm{kHz})$ and a 40x long working distance water objective. With this setup, docking and fusion of single vesicles can be monitored with an acquisition rate of 30 images per second. 


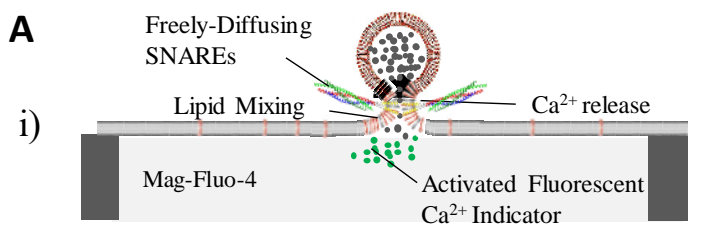

ii)

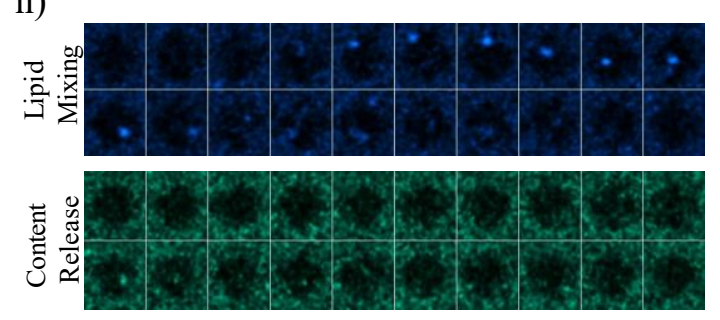

B

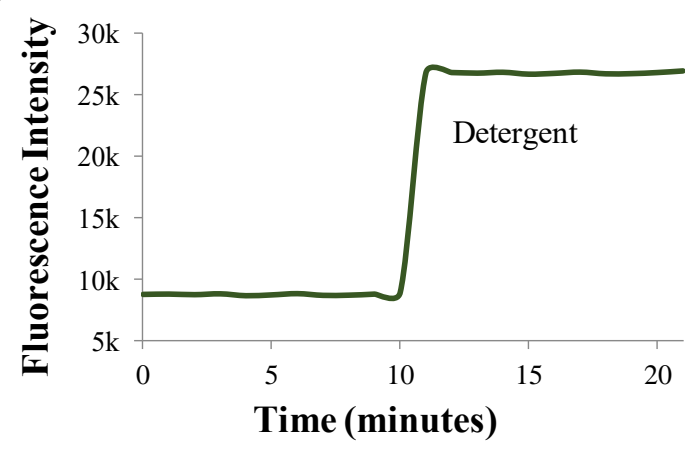

C

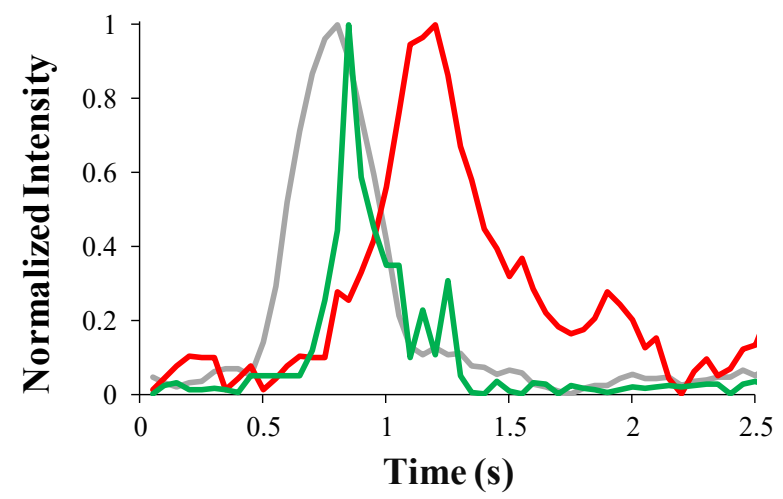

D

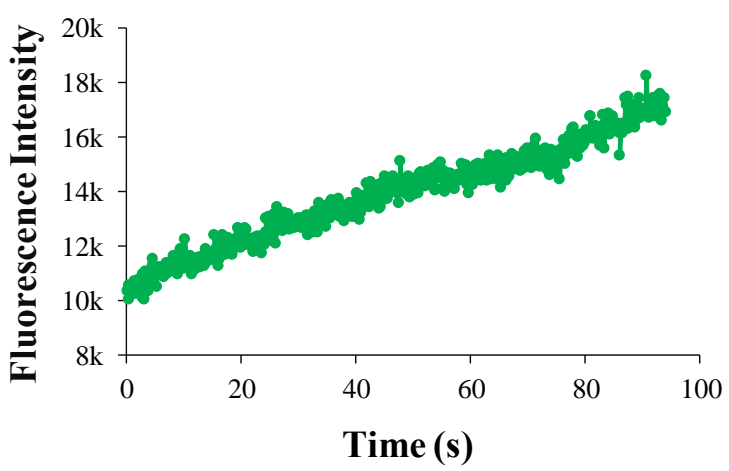

Figure 5. Calcium content Assay A) (i) Schematic of content release setup (ii) Liposomes were tracked using incorporated $1.5 \mathrm{~mol} \%$ of ATTO647N-DOPE. Image sequences of vesicle docked, diffuse on the membrane and fuse (colormap: Cyan Hot). Corresponding content release image sequences are shown below (green). An increase of the Mag-Fluo-4 fluorescence appears when the pore opens B) Efficiency of calcium trapping in the v-SUVs in bulk. Upon addition of 2.5\% dodecyl maltoside an increase of Mag-Fluo-4 fluorescence intensity due to the release of calcium ions from encapsulated liposomes can be observed. C) Fluorescence intensity of labelled lipids inside (grey) and outside (red) of the ROI and the calcium sensitive fluorophore (green) during fusion process. The content release happens during dequenching of the lipids in the v-SUV. D) Fluorescence intensity of calcium sensitive fluorophore Mag Fluor-4 over time during cumulative fusion events.

We have analyzed a total of $\sim 500 \mathrm{v}-S U V s$ that fused or remained docked for at least $600 \mathrm{~ms}$, $20 \%$ of them were obtained from experiments in which both lipid mixing and content release were monitored. Initially, all these v-SUVs displayed similar behaviors. They docked and freely diffused on the pore-spanning membrane. As expected from the mobility of the t-SNAREs, this 
diffusion remained confined above the hole of which $99 \%$ of the vesicles that came near the edge of the substrate bounced back to the center of the hole while the remaining $1 \%$ ended up being located near the substrate. After diffusing for approximately 5-8 frames (340-540 ms), the v-SUVs movement slowed down to the point that it looks almost immobile which may indicate an increased number of engaged SNARE complexes (each v-SUV contained 10 VAMP2 facing outside and potentially able to bind with a t-SNARE) (SI Movie 1, lipid). Then, the v-SUVs had two distinct fates: $\sim 60 \%$ of them exhibited full fusion (Fig. $4 \mathrm{~A}$ ) and $\sim 40 \%$ stayed docked on the membrane without fusion until their fluorescence was completely bleached (at which point they could no longer be monitored) (Figure 4B and Figure S3).

In lipid mixing experiments, fusion was attested by the sudden fluorescence increase of the ATTO647N dyes as a concentric spot followed with a burst. Then, as the lipids diffuse away, fluorescence decreased near the fusion spot. An elegant way to represent this migration of the lipids was proposed recently by Kuhlmann et al. ${ }^{9}$ Fluorescence is simultaneously monitored in a circular region of interest (ROI) encompassing the fusion spot (inner ROI) and in an annular ROI surrounding the circular one (outer ROI). When the fluorescent lipids left the circular ROI, they had to enter the annular one. Hence, a decrease of fluorescence intensity in the circular ROI must be followed by an increase of fluorescence in the annular one. This is exactly what we observed in our system (Figure 4A), demonstrating that these were actual fusion events.

It has been suggested that lipid mixing alone during membrane fusion experiments can sometimes lead to incorrect interpretation of the results because inner leaflet lipid dequenching is not always connected with the opening of the fusion pore and thus content release. ${ }^{12}$ Hence, 
simultaneously tracking lipid mixing and content release is critical to assert complete fusion actually occurred.

In our experiments, content release was monitored at the same vesicle position coordinates as the lipid mixing (Figure 5A). The kinetics of both processes can be compared (Figure 5C). Upon release of the encapsulated calcium in the v-SUVs a rapid increase of Mag- Fluo-4 fluorescence, which was captured beneath the pore spanning membrane, appeared concomitantly with the dequenching of the fluorophore of the lipids (see also SI Movie 1- content). This confirms that the cargo of the v-SUVs has crossed the pore-spanning membrane through a fusion pore. The timing of the Mag-Fluo-4 peak depends on the amount of calcium trapped in the liposomes, concentration of calcium sensitive fluorophore trapped below the membrane, and the temperature. Since calcium and Mag-Fluo-4 are trapped in the hole after fusion, the total Mag-Fluo-4 signal represents the cumulative fluorescence of all successive vesicles that released calcium into the hole. Figure 5D shows an example of the kinetics of this increase. The tight encapsulation of calcium inside the SUV was verified in bulk by measuring the fluorescence of Mag Fluor-4 before and after the addition of detergent (Figure 5B).

To check that the fusion process is driven only by the SNARE complex, we performed similar experiments with SUVs containing a v-SNARE mutant, VAMP2-4X, that only partially assembles with t-SNARE. ${ }^{18}$ Thus, these SUVs are expected to dock, but not fuse with the pore-spanning membrane. This is exactly what we observed. We recorded the docking of 200 SUVs containing VAMP2-4X. None of them underwent fusion. Instead, they all bleached after 100 secs (Figure 4 $\mathrm{B}$ and $\mathrm{C})$. Figure $4 \mathrm{C}$ shows the survival curve of docked liposomes on the pore-spanning membrane, only the liposomes containing wildtype v-SNAREs are fusing to the membrane 
whereas the 4x-mutant v-SNARE SUVs stayed docked and intact at the membrane. Moreover, it can be seen that about $50 \%$ of liposomes containing wildtype v-SNAREs fused within less than 2 seconds after docking which is expected for non-regulated fusion. Hence the fusion events we observed here are specifically driven by the SNARE complex with physiologically relevant kinetics. It should be noted that $\sim 36 \%$ of v-SUVs containing VAMP-4X were preferentially located near the rim (see SI movie 2).

\section{The Fusion Analyzer Software (FAS): Module for fast data analysis.}

Advanced software tools in combination with experimental method development often provide the researcher with fast and reliable data analysis in order to navigate through large volumes of data without missing any important information. In our experiments, manually analyzing hundreds of fusion events is time-consuming to a point that makes it almost impossible. Besides, to extract as much information as possible on the kinetics, the acquisition rate must be as fast as possible. The price to pay for this is a lower signal to noise ratio which makes the analysis even more complicated as it will require a clear assessment of the data quality. Hence, in addition to the development of the advanced proteo pore-spanning membrane system, we wrote a user friendly graphical interface software ("fusion analyzer", FAS) for automated detection of fusion events in both lipid mixing and content release experiments. This open source software is written using Matlab/C++ and gives access to multiple analysis modes. The data processing is performed in three steps, as shown in in figure 6A. The first component is a hole extraction module that applies a pattern recognition algorithm to detect the location and size of each hole and generates a pattern image in which the holes can be numbered to be processed and decrease computational cost by focusing on selected holes of interest. The second component is an image enhancement algorithm 
that is designed to improve the image quality, sharpening the edges of the fluorescence and reduce the noise. Silicon background reflection was removed by convoluting the original image with binary mask.

A

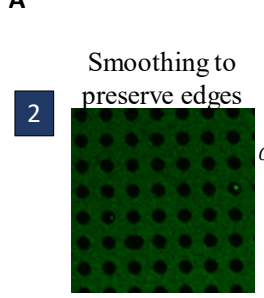

3

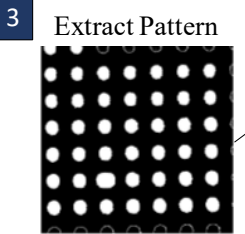

4

Hole Numbering

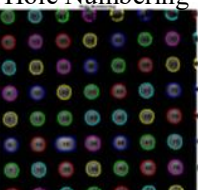

1

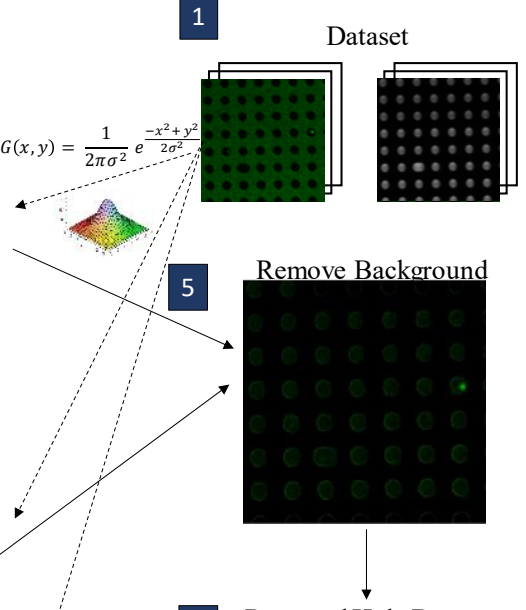

6

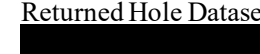

B
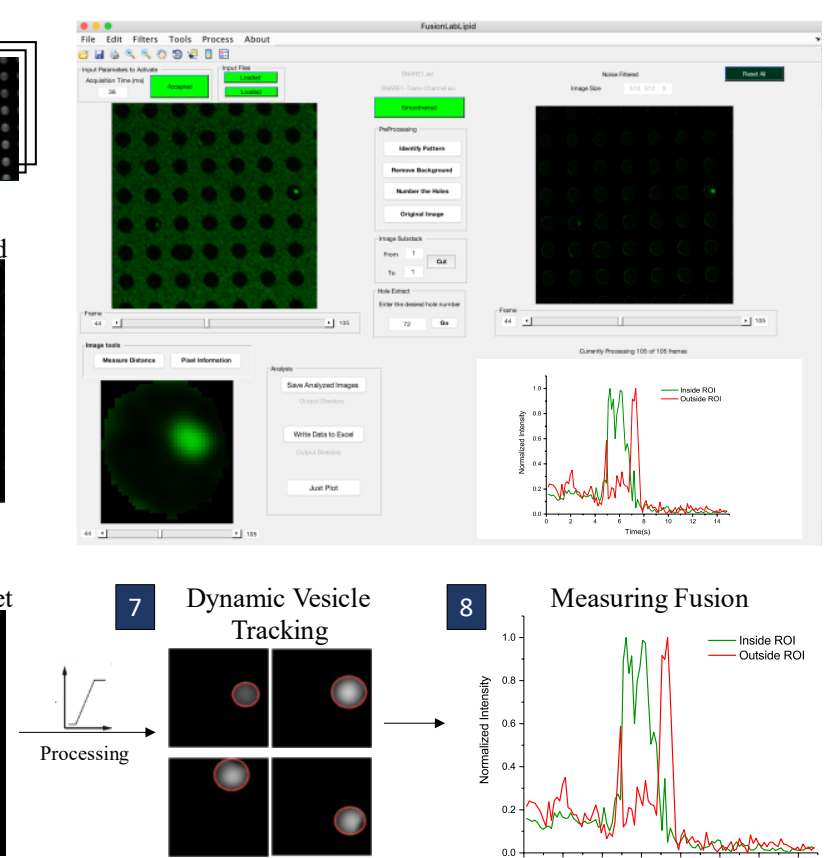

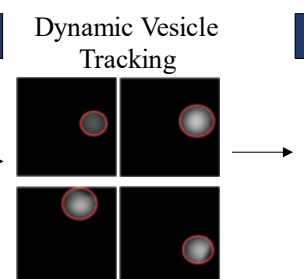

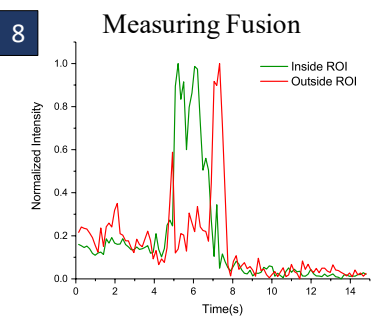

Figure 6. Fusion Analyzer Software (FAS). A) Diagram shows the steps involved in analysis. Movies from both fluorescence and transmission channel are imported to FAS (1) and convoluted with a Gaussian function to preserve object edges (2). Pattern recognition was employed to extract hole location and dimensions (3) and assigned a number to each hole for simplification and direct addressing (4). Background was removed by convoluting the mask obtained from pattern recognition (5). When the user chooses the desired hole to process, the program will extract the information of the particular hole (6), tracks the vesicles dynamically (7) and detect fusion events by measuring ROIs in each frame (8). B) FAS graphical user interface.

The final component is a dynamic tracking algorithm that extracts the information of vesicles in a space-time trajectory $(\mathrm{x}, \mathrm{y}, \mathrm{t})$ and analyzes fusion in a two dimensional (2-D) dataset. Centroid and Gaussian fit was employed to track the location of the vesicles with high accuracy. Dynamic 
ROI coordinates of the fluorescent lipid of the vesicle channel were used to track the fluorescence of the content- mixing channel at a given time. All of the image data analysis described in this paper has been done using this software (Figure 6B). The full source code of both lipid mixing and content release modes are provided with this work. Each component of the software can be easily customized according to research needs with minimal programing knowledge in MATLAB and $\mathrm{C}++$. For example, the pattern recognition feature in the software can be used to extract information of any grid geometry.

With regards to the data source, any imaging software can be used as our software works with avi-type movies as its primary source. In our case, we used avi-movies of 1000 frames (image size: 512 x 512 pixels) that have a size reasonable enough to perform the analysis on any laptop.

\section{DISCUSSION}

To date, there have been several setups used to study membrane proteins in model membrane systems. Supported membranes such as solid supported, tethered or polymer cushioned have been employed for a range of in vitro SNARE-mediated membrane fusion studies that allow for the detection of single vesicle fusion events, ${ }^{4,5,7}$ but have proven limited when it comes to the mobility of the incorporated proteins due to the interaction with the substrate. Pore-spanning membranes offer greater advantages over other established techniques as the reconstituted proteins can freely diffuse on the membrane and a second accessible aqueous compartment allowing this method likely to exhibit a more physiological environment.

Recently, pore-spanning membranes containing a t-SNARE $\Delta$ N49-complex were also used to study the fusion of LUVs containing v-SNAREs by lipid mixing. ${ }^{9}$ Our setup provides significant 
technical improvements. First, while lipid mixing is a common indicator of membrane fusion, we included a content release assay which is required to address the study of the full fusion process including the opening of the fusion pore. Second, we are using SUVs instead of LUV to better mimic the geometry of the synaptic vesicle. ${ }^{19}$ Finally, our preparation of the $\mathrm{Si} / \mathrm{SiO} 2$ chip is done by plasma cleaning followed by rinsing with ethanol, water and buffer making the system easy to establish. In the previous setup, the surface modification of the chip involves several non-trivial steps including coating with titanium, gold and n-propanolic 6-mercapto-1 hexanol to provide a monolayer surface, making it more challenging to apply. Both systems exhibit mobile proteins in the free-standing part of the bilayer. In our system, the protein density in the freestanding part of our membranes was stable for several hours.

Regarding the results of the fusion assay, our setup provided kinetics that are more physiologically relevant than any other setup. The time scale from the point the vesicle appeared in the frame and the disappearance of fluorescence signal after lipid mixing are broadly distributed between $\sim 600 \mathrm{~ms}$ and $4 \mathrm{~s}$. Most of the recorded docking and fusion events occur within the first 3 seconds with $\sim 50 \%$ of vesicles docking and fusing within 2 seconds. In comparison to the docking times and fusion rates of the other pore-spanning membrane fusion assay, ${ }^{9}$ only a few percent of vesicles were docked on the free-standing part of membrane and also exhibited long docking times ranging from 4 to $60 \mathrm{~s}$. These results are comparable to most established supported membrane studies exhibiting docking times in the range of tens of seconds. ${ }^{5,9,10,11,20}$ Another striking improvement of our setup is the high fraction of docked vesicles that actually fuse. We observed that $64 \%$ of the firmly docked vesicles ending up fusing. Conversely, previous studies presented lower percentage of fusion events (between 0.35 and 47\%)., ${ }^{5,10,11,20}$ Only one paper reported 
kinetics frequency of fusion close to ours. It used a supported membrane system that exhibited high percentage of fusion (50\%) and shorter times scale (less than $1 \mathrm{sec}$ ). ${ }^{7}$ This observation of faster docking times and a higher fusion rate compared to previous studies with freestanding membranes may be explained by the use of SUVs instead of LUVs. As the LUVs exhibit longer docking times they are more likely to get immobilized at the rim of the hole in the pore-spanning bilayers. This behavior of collecting near the rim has also been recorded in about $36 \%$ of the cases when using VAMP4X vesicles in our assay however this rarely occurred when using SUVs including wildtype v-SNAREs. The timescale of our recorded docking and fusion events is considered to be physiological relevant in most SNARE mediated fusion events. ${ }^{21}$

An interesting future extension of this work will be a faster acquisition speed and the incorporation of regulatory proteins such as synaptotagmin, Munc-13, Munc-18, and complexin. Overall, the study shows that dual detection (lipid and content release) method is a comprehensive measure to detect full fusion events. In addition, we have presented a new software, Fusion Analyzer (FAS), for measuring dynamic vesicle fusion to pore spanning membranes.

\section{MATERIALS AND METHODS}

Materials: The following materials were purchased and used without further purification: dioleoyl-phosphatidylcholine (DOPC), dioleoyl-phosphatidylserine (DOPS), dioleoylphosphoethanolamine-nitro-benzoxadiazolyl (NBD-PE), dioleoyl-glycero-phosphoethanolaminelissamine rhodamine B sulfonyl (LR-PE) (Avanti Polar Lipids, Alabaster, AL); dioleoylphosphoethanolamine-ATTO647N (DOPE-ATTO647N) (ATTO-Tec); $\alpha$-Hemolysin from 
Staphylococcus aureus, TCEP, HEPES, Trizma hydrochloride (TrisHCl), Sulforhodamine B sodium salt, calcium chloride, magnesium chloride, sodium chloride, ethanol, OptiPrep ${ }^{\mathrm{TM}}$ Density Gradient Medium Octyl- $\beta$-D-glucopyranoside (Sigma-Aldrich); Alexa Fluor ${ }^{\mathrm{TM}} 488$ C5 Maleimide, Texas Red ${ }^{\mathrm{TM}}$ C2 Maleimide, Slide-A-Lyzer ${ }^{\mathrm{TM}}$ Dialysis Cassettes (Fisher Scientific, Fair Lawn, NJ); Bio-Beads ${ }^{\mathrm{TM}}$ SM-2 Resin (Bio-Rad); 1,4-Dithiothreitol (DTT) (ROCHE); glass bottom petri dishes (Mattek); PreScission protease, a HiLoad Superdex 75 column and PD MidiTrap G-25 columns (GE Healthcare). $\mathrm{Si} / \mathrm{SiO}_{2}$ chips was purchased from Micromotive, Germany.

Proteins expression and purification: The full-length t-SNARE complex and Cys- t-SNARE complex (FLT-SNAP25B, Q20C) were derived from plasmid pTW34, and were expressed and purified as previously described. ${ }^{22,23}$ Cys free mouse VAMP2 (pet-SUMO-VAMP2, C103S) and Cys-VAMP2 (pet-SUMO-VAMP2, S28C, C103S) was derived from wild type VAMP2 (petSUMO-VAMP2) and were expressed and purified as previously described. ${ }^{24}$ Protein activity was verified after each protein preparation using t-SNARE, v-SNARE liposomes in a bulk fusion assay (Figure S1). ${ }^{1}$ In this bulk assay, the v-SNARE vesicles contained a quenched mixture of NBD and Rhodamine (1.5 mol\% each). Upon fusion with t-SNARE vesicles the fluorescent lipids dilute and a consequent increase in NBD fluorescence can be recorded. The mutant 4x VAMP2 (VAMP24X) was produced by cloning the full length, mouse VAMP2 into a pET SUMO vector that includes N-terminal 6x His tag, followed by site-directed mutation of L70D, A74R, A81D, and L84D and were expressed and purified as previously described. ${ }^{23}$ The cytosolic domain of the vSNARE (CVD) has been expressed and purified previously described. ${ }^{14}$ We used VAMP2-4X 
vesicles as a control containing half zippering 4x mutant v-SNAREs (Figure S2-B) As another control, FLT-liposomes were preincubated with CDV (the cytosolic domain of VAMP2, residues 1-94) preventing the docking of FLV-liposomes. In the bulk assay, we found that, as expected, only v-SNARE vesicles progress to fusion, whereas experiments with VAMP2-4X and CDV vesicles did not display any significant fusion (Figure S2-C).

Proteins labeling: The transmembrane t-SNARE Q20C was labeled with Alexa Fluor® similarly to previous reports. ${ }^{14}$ The protein was first reduced by incubating with $4 \mathrm{mM}$ TCEP for 30 minutes at $4{ }^{\circ} \mathrm{C}$ with gentle rotation and centrifuged at $14,000 \mathrm{rpm}$ for 20 minutes at $4{ }^{\circ} \mathrm{C}$ to remove any precipitation. Fluorescence dye was added into the protein solution at a molar ratio of 3:1 and the mixture were incubated for 1 to 2 hours at room temperature with gentle rotation. Unreacted dye was removed by passing through a PD MidiTrap G-25 column (GE Healthcare) three times.

Proteoliposomes Reconstitution: SNARE proteins were reconstituted into liposomes using rapid detergent dilution and dialysis as previously described ${ }^{25}$ with slight modifications. Previous studies have shown that rapid dilution produces a narrow distribution of liposomes similar to the size of synaptic vesicles $(30-50 \mathrm{~nm}) .{ }^{26}$ The small vesicle diameter is crucial for measuring in vitro fusion, as these vesicles possess a high curvature stress making it more fusogenic than larger vesicles. ${ }^{19}$ t-SNARE SUVs: Lipids (DOPC/DOPS 70: $30 \mathrm{~mol} \%$ ) were dried under nitrogen and vacuum and then rehydrated with buffer (125 mM HEPES; $500 \mathrm{mM} \mathrm{KCl;} 5 \mathrm{mM}$ DTT, pH 7.4) containing $1 \%$ n-octyl- $\beta$-D-glucoside (OG) with a final protein: lipid ratio of 1:1000. After 20 min of shaking, rapid dilution was done by addition of 2 times buffer to dilute the detergent concentration below 
the critical micellar concentration and mixed for another $20 \mathrm{~min}$. The resulting proteo-liposomes (final lipid concentration: $3 \mathrm{mM}$ ) were dialyzed overnight in buffer with $4 \%(\mathrm{w} / \mathrm{v})$ of SM2Biobeads, using dialysis cassette with a molecular weight cutoff of 7,000 Da.

VAMP2 and VAMP2-4X SUVs: Liposomes for single molecule measurements were made of 83 mol \% DOPC, 15 mol \% DOPS, and 2 mol\% DOPE-ATTO-647N with a final protein: lipid ratio of 1:400. Dried lipids were rehydrated with buffer (25 mM HEPES, $100 \mathrm{mM} \mathrm{KCl,} \mathrm{1mM} \mathrm{DTT,} \mathrm{pH}$ 7.4) with $2 \%$ OG and shaken vigorously for 20 mins. The mixture was diluted 4 times to $1 \mathrm{ml}$ using detergent-free buffer, then dialyzed overnight supplemented with 4\% (w/v) SM-2 Biobeads using a 7000 Da cut off dialysis cassette. Dialyzed samples were mixed 1:1 (v/v) with 60\% Optiprep and layered below 20\% and 0\% Optiprep in buffer. After centrifugation in a Beckman SW41 Ti rotor at $40,000 \mathrm{rpm}, 4-5 \mathrm{hr}$ at $4{ }^{\circ} \mathrm{C}, 400 \mu \mathrm{l}$ of liposomes were recovered at the $0 \%-20 \%$ interface. The same procedure was employed for the reconstitution of VAMP2-4X liposomes. $\boldsymbol{C a}^{2+}$ loaded SUVs: Calcium (50 mM) encapsulated liposomes containing v-SNAREs (protein: lipid ratio: 1:400) were prepared as previously described ${ }^{27}$ with small changes. Atto-647N-DOPE was used as a lipid marker and v-SNAREs proteins were incorporated into liposomes. Freshly prepared liposomes were checked for calcium encapsulation by recording the fluorescence of the liposome sample on the plate reader in the presence of $2 \mu \mathrm{M}$ Mag-Fluo- 4 for $30 \mathrm{~min}$. If the signal remained stable, $10 \mu \mathrm{L}$ of $2.5 \%$ dodecyl maltoside was added to the sample and a fluorescence increase of Mag Fluor4 was recorded due to the release of calcium ions. In this study, we only used liposomes in the single vesicle assay which showed no calcium leaking in the plate reader for at least 30 minutes. Calcium loaded liposomes were always made fresh before fusion experiment. 
t-SNARE-GUV formation by osmotic shock: GUVs containing t-SNAREs were prepared as previously described. ${ }^{14}$ Briefly, the coverslip glass was cleaned with isopropanol and a dust free tissue and dried with pressurized Argon. A $2 \mu 1$ drop of small liposomes solution was placed on the coverslip and dried at room temperature under atmospheric pressure. Then, it was rehydrated with $6 \mu$ deionized water, and dried again under the same conditions. In the next step the lipid film is rehydrated once more with a larger deionized water volume of $10 \mu 1$ for 45 mins. GUVs grew instantly from the rehydrated lipid films mainly in the areas with high concentration of lipids resulting in a buffer concentration of $25 \mathrm{mM}$ HEPES, $100 \mathrm{mM} \mathrm{KCl,} \mathrm{1mM} \mathrm{DTT,} \mathrm{pH} \mathrm{7.4.}$

Generation of free standing lipid bilayer: $\mathrm{Si} / \mathrm{SiO}_{2}$ chips containing an array of 5 um holes (Micromotive, Muenster, Germany) were glued with optically clear adhesive (Norland Optical Adhesives, NOA 81) to an eight- well sample holders (Ibidi sticky slide cat. no. 80828). The chips were cleaned using plasma for 4 mins. To prevent air bubbles in the holes the chips were incubated for $5 \min$ in $300 \mu \mathrm{L}$ ethanol on a shaker, followed by 5 times $300 \mu \mathrm{L}$ rinse with water. It is important that the chips do not run dry because air bubbles could possibly form in the holes. The water is exchanged through a 5 times rinse with $300 \mu \mathrm{L}$ of $5 \mathrm{mM}$ magnesium chloride $\left(\mathrm{MgCl}_{2}\right)$ containing buffer (25 mM HEPES, $100 \mathrm{mM} \mathrm{KCl,} \mathrm{1mM} \mathrm{DTT,} \mathrm{pH} \mathrm{7.4).} \mathrm{The} \mathrm{bilayers} \mathrm{prepared} \mathrm{for}$ content-release experiments had $1 \mathrm{mM} \mathrm{MgCl} 2$ to reduce $\mathrm{Mg}^{2+}$ ions interactions with fluorophores trapped below bilayer. $2 \times 10 \mu \mathrm{L}$ of preformed proteo-GUVs was pipetted onto the chip with a halfcut pipette tip. The GUVs were allowed to settle for about 20 minutes and centrifuged in a microplate holder for $5 \mathrm{~min}$ at $100 \mathrm{x} \mathrm{g}$. Non-spread GUVs and the magnesium chloride are then removed by a final 5 times rinse with $300 \mu$ l magnesium free reconstitution buffer. Same 
osmolarity was maintained on both sides of the membrane. Lamelarity of the membrane was tested using $0.5 \mu \mathrm{M} \alpha$-hemolysin. Sealing of the pore-spanning membranes was studied by first capturing $20 \mathrm{mM}$ TRITC beneath membrane followed by with $20 \%$ SDS.

Fusion assay: In order to record membrane fusion v-SUVs (600 nM lipid) were added to the pore spanning membrane containing Alexa488-labeled t-SNAREs in $25 \mathrm{mM}$ HEPES, $100 \mathrm{mM} \mathrm{KCl,}$ 1mM DTT, pH 7.4 at $37{ }^{\circ} \mathrm{C}$. Fusion was followed by lipid mixing (tracking 3 mol\% Atto647NDOPE in V-SUVs) and content release. In the latter setup, calcium was captured in the v-SUVs whereas buffer containing a calcium-activated fluorophore, $20 \mu \mathrm{M}$ Mag-Fluo-4 (K $\mathrm{K}_{\mathrm{d}}$ for calcium: $22 \mu \mathrm{M})$, was trapped below the pore-spanning membrane.

Fluorescence microscopy: An inverted Laser-scanning confocal microscope (Leica-SP5) equipped with multi-wavelength Argon Laser including 488, and diode lasers (532 and $641 \mathrm{~nm}$ ), and a long- working distance $43 \mathrm{X}$ water immersion objective (NA 1.1) was used to image the $\mathrm{Si} / \mathrm{SiO}_{2}$ chip and to follow membrane fusion. The emission light was spectrally separated and collected by photo multiplier tubes. A resonant scanner was used to acquire movies at a speed of $36 \mathrm{~ms}$ per frame.

Image dataset and Hole Extraction: The software "Fusion Analyzer" was developed in MATLAB/ C++ IDE and tested on image datasets acquired with a Leica Scanning Confocal microscope. Images of fluorescent lipid, vesicle and the transmission light channel were acquired at a speed of $96 \mathrm{~ms} /$ frame for a total of 1000 frames per movie for convenient export as an avi 
movie format file from the LAS AF software. The Fusion Analyzer independently imports each channel file in avi format. The transmission light channel movie is used for pattern recognition of the holes for easier processing. The extracted pattern is used to create a binary mask. The mask is overlaid with the original dataset to remove the reflection from silicon surface. Numbers were assigned to each hole for identification and simplified data analysis. The desired hole from the image dataset is extracted for vesicle fusion analysis.

Vesicle Detection and Tracking: For accurate vesicle detection, the desired hole image dataset is convoluted with a Gaussian beam point spread function (Eq.1). This convolution process sets a new value to each pixel based on the weighted average of that pixel's neighborhood. The original pixel's value receives the heaviest weight and neighboring pixels receive smaller weights, which creates a blur that preserves edges of the vesicles. In this case, each image in the dataset is smoothened with 2-D Gaussian smoothing kernel with standard deviation of $2(\sigma=2)$.

$$
G(x, y)=\frac{1}{2 \pi \sigma^{2}} e^{\frac{-\left(x^{2}+y^{2}\right)}{2 \sigma^{2}}}
$$

Here, $\mathrm{x}$ is the distance from the origin in the horizontal axis, $\mathrm{y}$ is the distance from the origin in the vertical axis, and $\sigma$ is the standard deviation of the Gaussian distribution.

Further, the smoothened dataset is filtered with median filter to sharpen the image by having a median value in a 3-by-3 size around the corresponding pixel in the input image. A binary mask for every frame in the dataset is created with the value above the threshold. The values below the threshold are assumed to be zero, whereas those above threshold are unaltered. An 8-connectedcomponent labeling method is employed to identify the objects that have minimum eight point connected regions in the frame, which is ideal to get rid of a few pixels noise. We consider the 
objects as vesicles if it has an area above thresholding, which is 40 pixels in our case for a $40 \mathrm{X}$ water immersion objective with $\mathrm{NA}=1.1$. As the area of the vesicles depends on the objective magnification, these numbers can be adjusted to get accurate tracking. Properties of the vesicles such as area and location were measured. The location of the vesicles can be traced by repeating the above procedure for every frame in the dataset. Generally, algorithms that track single particles either depend on finding the centroid and fitting Gaussian curves to the intensity or crosscorrelation. We chose a simple and efficient method to estimate the absolute positions in each image independently by calculating the centroid of two sequential images of a vesicle for calculating the distance vesicle moved. We assume I is the intensity of an image with object and background. Eq. 2 gives the centroid calculation for a single axis:

$$
\begin{gathered}
C_{x}=\sum_{i=1}^{n} \sum_{j=1}^{m}\left(x_{i} . I_{i j}\right) / \sum_{i=1}^{n} \sum_{j=1}^{m} I_{i j} \\
C_{y}=\sum_{i=1}^{n} \sum_{j=1}^{m}\left(y_{i} I_{i j}\right) / \sum_{i=1}^{n} \sum_{j=1}^{m} I_{i j}
\end{gathered}
$$

where $x_{i}$ is the coordinate on the $x$ axis, $y_{i}$ is the coordinate on the $y$ axis and $\mathrm{I}_{\mathrm{ij}}$ is the intensity of the pixel. Centroids are calculated for one image and subtracted for a successive image to determine the distance of the object moved. These numbers are useful to extract the diffusion coefficient of the particle and track the movement of the vesicle itself.

Analyzing Membrane Fusion: Our newly developed open source software "Fusion Analyzer" is an advanced platform to simplify the analysis of measuring fusion to free-standing lipid membranes. To track membrane fusion events, in each frame two region of interest (ROI) were drawn on the image of the freestanding membrane, one that tracks the vesicle movement and the 
other one drawn outside the vesicle ROI. When no vesicle could be found, both ROI were drawn at the center of image $(x / 2, y / 2)$. In the event of fusion, the fluorescent lipids from the vesicle spread from the vesicle ROI to the outer ROI, which increases the fluorescence intensity in the outer annular ROI channel. Based on the intensity remaining in the vesicle ROI and outer annular ROI, the software can predict the stages of fusion such as docking, hemi and full fusion. A user manual of the program and sample data is available at the website for download.

\section{ASSOCIATED CONTENT}

\section{Supporting Information}

The Supporting Information is available free of charge on the ACS Publications website.

Fusion Analyzer Software (FAS) MATLAB Source-code: All the functions required to run both lipid and content mixing analysis can be found in the file. (PDF) The files can also be downloaded from Matlab Central "https://www.mathworks.com/matlabcentral/fileexchange/66521-fusionanalyzer--fas"

Movie 1: Movies showing docking and fusion event of v-SUVs by lipid mixing (ATTO647NDOPE, movie 1 -lipid) and content release (Mag-Fluo-4, movie 1 - content) to a freestanding bilayer containing t-SNAREs cropped from a typical larger movie acquired using Leica confocal scanning microscope (LSCM) at a speed of 27 frames/s. This movie is in avi format that can be opened with ImageJ. (Size of hole: $5 \mu \mathrm{m}$ ) (AVI )

Movie 2: Movie showing VAMP2-4X vesicle (Atto647N-DOPE) dock on the freestanding membrane containing t-SNAREs without proceeding to fusion. The movies were acquired in LSCM at a speed of 2.6 frames/s. This movie is in avi format that can be opened with ImageJ. (Size of hole: $5 \mu \mathrm{m})(\mathrm{AVI})$ 


\section{AUTHOR INFORMATION}

\section{Corresponding Author}

*Correspondence to Frederic Pincet (pincet@lps.ens.fr) or James E. Rothman (james.rothman@yale.edu)

\section{Present Addresses}

$\dagger$ Drug Discovery Unit, Cancer Research UK Beatson Institute, Garscube Estate, Switchback Road, Glasgow G61 1BD, UK.

\section{Author Contributions}

The manuscript was written through contributions of all authors. / All authors have given approval to the final version of the manuscript. $\$$ S.R and A.G contributed equally.

\section{Funding Sources}

This work was supported by Agence Nationale de la Recherche (ANR) ANR-14-1CHN-0022-01 grant

\section{ACKNOWLEDGMENTS}

We thank Dr Ilja Kuester (Havard Medical School, USA) for inspiring discussions regarding the formation of freestanding membranes and Joel Lemiere (Yale University) for very useful discussions regarding the alpha-hemolysin experiments. We also thank Kirill Grushin (Yale University, USA) for providing the SNARE protein pictures. 


\section{REFERENCES}

1. Weber, T.; Zemelman, B. V.; McNew, J. A.; Westermann, B.; Gmachl, M.; Parlati, F.; Sollner, T. H.; Rothman, J. E. SNAREpins: minimal machinery for membrane fusion. Cell 1998, 92 (6), 759-72.

2. Yoon, T. Y.; Okumus, B.; Zhang, F.; Shin, Y. K.; Ha, T. Multiple intermediates in SNAREinduced membrane fusion. Proc Natl Acad Sci U S A 2006, 103 (52), 19731-6.

3. Kyoung, M.; Zhang, Y.; Diao, J.; Chu, S.; Brunger, A. T. Studying calcium-triggered vesicle fusion in a single vesicle-vesicle content and lipid-mixing system. Nat Protoc 2013, 8 (1), $1-16$.

4. Domanska, M. K.; Kiessling, V.; Stein, A.; Fasshauer, D.; Tamm, L. K. Single vesicle millisecond fusion kinetics reveals number of SNARE complexes optimal for fast SNAREmediated membrane fusion. J Biol Chem 2009, 284 (46), 32158-66.

5. Fix, M.; Melia, T. J.; Jaiswal, J. K.; Rappoport, J. Z.; You, D.; Sollner, T. H.; Rothman, J. E.; Simon, S. M. Imaging single membrane fusion events mediated by SNARE proteins. Proc Natl Acad Sci U S A 2004, 101 (19), 7311-6.

6. Kiessling, V.; Domanska, M. K.; Tamm, L. K. Single SNARE-mediated vesicle fusion observed in vitro by polarized TIRFM. Biophys J 2010, 99 (12), 4047-55.

7. Karatekin, E.; Di Giovanni, J.; Iborra, C.; Coleman, J.; O'Shaughnessy, B.; Seagar, M.; Rothman, J. E. A fast, single-vesicle fusion assay mimics physiological SNARE requirements. Proc Natl Acad Sci U S A 2010, 107 (8), 3517-21.

8. Schwenen, L. L.; Hubrich, R.; Milovanovic, D.; Geil, B.; Yang, J.; Kros, A.; Jahn, R.; Steinem, C. Resolving single membrane fusion events on planar pore-spanning membranes. Sci Rep 2015, 5, 12006.

9. Kuhlmann, J. W.; Junius, M.; Diederichsen, U.; Steinem, C. SNARE-Mediated SingleVesicle Fusion Events with Supported and Freestanding Lipid Membranes. Biophys J 2017, 112 (11), 2348-2356. 
10. Bowen, M. E.; Weninger, K.; Brunger, A. T.; Chu, S. Single molecule observation of liposome-bilayer fusion thermally induced by soluble N-ethyl maleimide sensitive-factor attachment protein receptors (SNAREs). Biophysical Journal 2004, 87 (5), 3569-3584.

11. Xu, W. M.; Nathwani, B.; Lin, C. X.; Wan, J.; Karatekin, E.; Pincet, F.; Shih, W.; Rothman, J. E. A Programmable DNA Origami Platform to Organize SNAREs for Membrane Fusion. Journal of the American Chemical Society 2016, 138 (13), 4439-4447.

12. Chan, Y. H.; van Lengerich, B.; Boxer, S. G. Effects of linker sequences on vesicle fusion mediated by lipid-anchored DNA oligonucleotides. Proc Natl Acad Sci U S A 2009, 106 (4), 979-84.

13. Smith, M. B.; Karatekin, E.; Gohlke, A.; Mizuno, H.; Watanabe, N.; Vavylonis, D. Interactive, Computer-Assisted Tracking of Speckle Trajectories in Fluorescence Microscopy: Application to Actin Polymerization and Membrane Fusion. Biophysical Journal 2011, 101 (7), 1794-1804.

14. Motta, I.; Gohlke, A.; Adrien, V.; Li, F.; Gardavot, H.; Rothman, J. E.; Pincet, F. Formation of Giant Unilamellar Proteo-Liposomes by Osmotic Shock. Langmuir : the ACS journal of surfaces and colloids 2015, 31 (25), 7091-9.

15. Song, L.; Hobaugh, M. R.; Shustak, C.; Cheley, S.; Bayley, H.; Gouaux, J. E. Structure of staphylococcal alpha-hemolysin, a heptameric transmembrane pore. Science 1996, 274 (5294), 1859-66.

16. Ma, L.; Cockroft, S. L. Biological nanopores for single-molecule biophysics. Chembiochem 2010, $11(1), 25-34$.

17. Kusters, I.; van Oijen, A. M.; Driessen, A. J. Membrane-on-a-chip: microstructured silicon/silicon-dioxide chips for high-throughput screening of membrane transport and viral membrane fusion. Acs Nano 2014, 8 (4), 3380-92.

18. Krishnakumar, S. S.; Radoff, D. T.; Kummel, D.; Giraudo, C. G.; Li, F.; Khandan, L.; Baguley, S. W.; Coleman, J.; Reinisch, K. M.; Pincet, F.; Rothman, J. E. A conformational switch in complexin is required for synaptotagmin to trigger synaptic fusion. Nat Struct Mol Biol 2011, 18 (8), 934-40.

19. Bentz, J.; Duzgunes, N. Fusogenic capacities of divalent cations and effect of liposome size. Biochemistry 1985, 24 (20), 5436-43. 
20. Schwenen, L. L. G.; Hubrich, R.; Milovanovic, D.; Geil, B.; Yang, J.; Kros, A.; Jahn, R.; Steinem, C. Resolving single membrane fusion events on planar pore-spanning membranes. Scientific reports 2015, 5, 12006.

21. Kasai, H.; Takahashi, N.; Tokumaru, H. Distinct Initial Snare Configurations Underlying the Diversity of Exocytosis. Physiol Rev 2012, 92 (4), 1915-1964.

22. Li, F.; Kummel, D.; Coleman, J.; Reinisch, K. M.; Rothman, J. E.; Pincet, F. A half-zippered SNARE complex represents a functional intermediate in membrane fusion. Journal of the American Chemical Society 2014, 136 (9), 3456-64.

23. Li, F.; Tiwari, N.; Rothman, J. E.; Pincet, F. Kinetic barriers to SNAREpin assembly in the regulation of membrane docking/priming and fusion. Proc Natl Acad Sci U S A 2016, 113 (38), 10536-41.

24. Shen, J. S.; Tareste, D. C.; Paumet, F.; Rothman, J. E.; Melia, T. J. Selective activation of cognate SNAREpins by Sec1/Munc18 proteins. Cell 2007, 128 (1), 183-195.

25. Scott, B. L.; Van Komen, J. S.; Liu, S.; Weber, T.; Melia, T. J.; McNew, J. A. Liposome fusion assay to monitor intracellular membrane fusion machines. Methods Enzymol 2003, 372, 274-300.

26. Ji, H.; Coleman, J.; Yang, R.; Melia, T. J.; Rothman, J. E.; Tareste, D. Protein Determinants of SNARE-Mediated Lipid Mixing. Biophysical Journal 2010, 99 (2), 553-560.

27. Shi, L.; Howan, K.; Shen, Q. T.; Wang, Y. J.; Rothman, J. E.; Pincet, F. Preparation and characterization of SNARE-containing nanodiscs and direct study of cargo release through fusion pores. Nat Protoc 2013, 8 (5), 935-948. 


\section{$\underline{\text { Table of Content Graphic }}$}

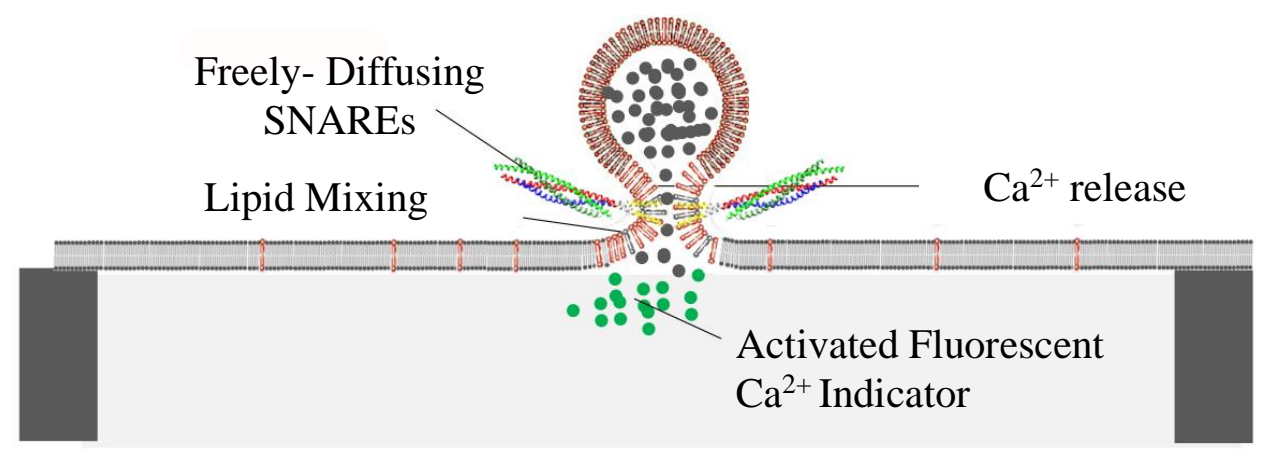

